\title{
Biologic effects of radiofrequency thermal ablation on non-small cell lung cancer: Results of a pilot study
}

\author{
Marcello Carlo Ambrogi, MD, ${ }^{a}$ Gabriella Fontanini, MD, ${ }^{\mathrm{b}}$ Roberto Cioni, MD, ${ }^{\mathrm{c}}$ Pinuccia Faviana, MD, \\ Olivia Fanucchi, MD, and Alfredo Mussi, MD ${ }^{a}$
}

Objectives: Radiofrequency ablation of lung tumors is an emerging technique with promising results. To achieve more information on its effects on pulmonary parenchyma and primary tumors, and to assess its efficacy in determining complete necrosis of the lesion, we led a pilot study consisting of thermal ablation followed by surgical resection.

Methods: Ten patients with early stage non-small cell lung cancer were enrolled in the study. In 5 patients radiofrequency ablation was achieved through thoracotomy just before the surgical resection. In another 5 patients radiofrequency ablation was performed percutaneously, under computed tomography guidance, and the surgical resection was performed after 15 days.

Results: Nine of the 10 patients enrolled in the study were available for analysis. There were 8 men and 1 woman with a mean age of 65.5 years. In all cases histologic diagnosis was available before radiofrequency ablation; adenocarcinoma was identified in 5 patients, and squamous cell carcinoma was identified in 4 patients. Microscopic examination showed complete necrosis of the tumor in 6 of 9 cases. No anatomopathologic alterations were seen in surrounding distant parenchyma.

Conclusions: Our study confirms the safety of radiofrequency ablation, particularly underlining the possibility of performing a thermal lesion in the lung in a controlled manner. It also assesses that radiofrequency ablation may be effective in the local control of primary lung cancer. However, surgery still represents the mainstay of treatment of non-small cell lung cancer, and radiofrequency ablation must be reserved for high-risk patients.

From the aThoracic Surgery, Cardiac and Thoracic Department; ' $P$ Pathology, Department of Oncology Transplants and New Technologies, ${ }^{\circ}$ Radiology, Department of Oncology Transplants and New Technologies, Pisa, Italy.

Received for publication Sept 6, 2005; accepted for publication Dec 30, 2005.

Address for reprints: Marcello Carlo Ambrogi, MD, Cardiac and Thoracic Department, via Paradisa 2, 56124, Pisa, Italy (E-mail: m.ambrogi@med.unipi.it).

J Thorac Cardiovasc Surg 2006;131:1002-6 $0022-5223 / \$ 32.00$

Copyright $(\odot) 2006$ by The American Association for Thoracic Surgery

doi:10.1016/j.jtcvs.2005.12.040
$\mathrm{T}$ he results of the present modalities of treatment of non-small cell lung cancer (NSCLC) are poor in all but stage I disease. ${ }^{1}$ Surgery still represents the treatment with the greatest chance of cure in case of localized disease (stages I, II, and IIIa); nevertheless, surgical treatment is not always feasible because of medical contraindications or refusal by the patient. In this case, chemotherapy and radiotherapy, alone or in combination, give a modest therapeutic contribution, primarily the palliative sort. $^{2}$

In this scenario it is understandable that minimally invasive treatments often receive great interest, as is now happening for radiofrequency thermal ablation (RFA). This method has been successfully used for the treatment of many solid tumors. $^{3-7}$ Thin, metallic probes, similar to aspiration biopsy needles, are percutaneously inserted into the lesion using computed tomography (CT) scanning or ultrasound guidance. Radiofrequency energy is then applied to achieve a temperature greater than $60^{\circ} \mathrm{C}$ (in most cases $90^{\circ} \mathrm{C}$ ). Thus, coagulative necrosis of the tumor is induced in a controlled manner.

More recently this technique has been applied to pulmonary tumors, showing promising preliminary results. ${ }^{8-11}$ All of the recently reported clinical trials analyzed 


\section{Abbreviations and Acronyms \\ $\mathrm{CT}=$ computed tomography \\ $\mathrm{NADH}=$ reduced nicotinamide adenine dinucleotide \\ NSCLC $=$ non-small cell lung cancer \\ RFA $=$ radiofrequency ablation}

the results of the procedure from a radiologic point of view. However, little is still understood about the biologic effects of RFA on the human lung and its tumors.

After an initial experience using an animal model, ${ }^{12}$ we transferred this technique to human lung tumors. The object of this articles concerns the results of 2 "ablate and resect" protocols, which provided for radiofrequency ablation of the tumor followed by surgical resection. To our knowledge this is the largest single institutional series.

\section{Materials and Methods}

This study was conducted with the approval of the local ethical committee for human research care.

\section{Radiofrequency Generator and Devices}

We used a generator able to provide monopolar radiofrequency energy to perform coagulation and ablation of soft tissue (Model 1500 , RITA Medical System, Mountain View, Calif). This is an automatic apparatus with a maximum power output of $150 \mathrm{~W}$ (operating at $460 \mathrm{~Hz}$ ). It has multiple temperature displays as well as impedance and power monitoring. The energy was transferred into the tissue by means of a disposable array (StarBurst XL, RITA Medical System). It consists of a 15-gauge needle with 9 deployable electrodes that open in a flower-like manner up to $5 \mathrm{~cm}$ (Figure 1). Five electrodes are equipped with a thermocouple to continuously measure the temperature inside the tissue. Two dispersive electrode pads were applied to each shaved leg to ground the current and to reduce risks of skin heat injuries. Once the system was powered up, the physician set the parameters of the ablation: the mode of operation (we used "average temperature mode," in which delivered power was automatically regulated to maintain the target temperature set); target temperature; ablation time at target temperature; and maximum power delivery level, which can be modified at any time during the procedure.

\section{Entry Criteria}

Patients were required to meet the following specific criteria for enrollment, in addition to those standard to tolerate major pulmonary resection: at least 18 years of age; preoperative histologic proof of the tumor; clinical stage I and II NSCLC (assessed by CT scan); maximum diameter of the tumor less than or equal to 3.5 $\mathrm{cm}$, and farther than $1 \mathrm{~cm}$ from major blood vessels or airways; and signed informed consent.

Exclusion criteria included patients aged more than 75 years, with pleural effusion, or who required a resection greater than a lobectomy.

\section{Radiofrequency Ablation Procedure and Surgical Resection}

In the first protocol, RFA was performed during open surgery. With the patient under general anesthesia and selective intubation, a thoracotomy was performed to allow surgical resection. The lobar hilum was isolated to allow quick surgical resection in case of complications, but blood supply and ventilation were preserved to reproduce better physiologic conditions. The needle was inserted into the tumor by digital palpation guidance, and the electrodes were deployed with the lung deflated to permit better facility for correctly placement of the device into the lesion. The generator was powered up and radiofrequency ablation was performed with the lung ventilated again; standard vital signs of the patients were recorded. At the end of the procedure, the electrodes were retracted and the needle was withdrawn slowly with a rotatory motion. A lobectomy with mediastinal lymph node dissection was then completed as planned. RFA and surgical resection were always performed by the same surgeons (MCA and AM, respectively).

In the second protocol, the patients underwent percutaneous RFA by CT guidance, under local anesthesia and conscious sedation. Surgical resection was delayed 15 days after the thermal ablation and a lobectomy, with mediastinal lymph node dissection, performed in all cases. In these patients, RFA was always performed by a surgeon (MCA) and radiologist (RC), and the surgical resection was performed by the same surgeon (AM).

In all cases the target temperature was $90^{\circ} \mathrm{C}$, which was maintained for 15 to 27 minutes according to the size of the tumor and, consequently, the deployment of the electrodes. When technically possible, we always pursued the objective of encompassing the neoplastic lesion with a thermal one of at least $1 \mathrm{~cm}$ larger.

At the end of the surgical resection, all specimens were processed for pathologic evaluation.

\section{Anatomopathologic Study}

The specimens were sectioned across the maximum diameter, and macroscopic findings were recorded. They were preserved in a formalin solution and immediately transferred to our pathology unit for microscopic analysis, which was performed on average 36 hours after resection by 2 highly qualified pathologists with consolidated experience on lung cancer (GF and PF). The protocol provides that the specimen is fixed in $40 \%$ phosphate-buffered formalin and that the thermal lesion (including the tumor) is divided into 3 to 4 blocks (according to the size of the lesion). Each block is cut at intervals of $30 \mu \mathrm{m}$ (levels). For each level, 2 sections $(5 \mu \mathrm{m})$ are obtained by microtome, all of which are routinely stained for histologic examination using alternately hematoxylin-eosin staining. The sections were examined by both pathologists using a light microscope with low $(4-10 \times)$ and high $(40 \times)$ zoom lenses. Multiple samplings were made around the treated area: Two samples were taken $1 \mathrm{~cm}$ distant from the treated area, 2 samples were taken $2 \mathrm{~cm}$ distant from the treated area, and 2 samples were taken in the surrounding parenchyma, far from the thermal lesion.

\section{Results}

Ten patients were enrolled in this study, but in 1 case the patient refused surgical resection after percutaneous RFA, and therefore was not available for analysis. Of the remain- 


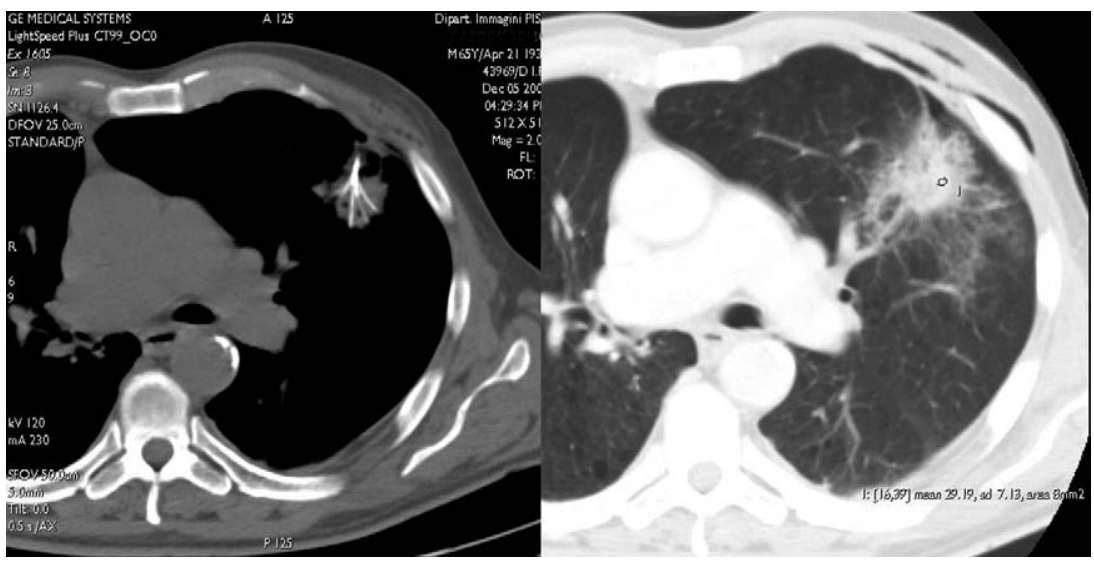

Figure 1. CT images during and just after percutaneous radiofrequency ablation of an NSCLC. It is to observe the ground-glass density area of the thermal lesion encompassing the neoplastic one.

ing 9 patients, 8 were male and 1 was female with a mean age of 65.5 years (range 55-70 years). In all cases histologic diagnosis was available before RFA; we identified adenocarcinoma in 5 patients and squamous cell carcinoma in 4 patients. All lesions were peripheral with a mean size of 2.8 $\mathrm{cm}$ (range 1.5-3.5). Postoperative pathologic staging was stage $I$ in 6 cases and stage II in 3 cases. Overall time for the entire ablation was 24 minutes on average (range 18-33 minutes). This includes the time of RFA at target temperature (19 minutes on average) and the time to reach the target temperature ( 5 minutes on average), at start and every time it was lost after the serial expansion of the electrodes.

No complications related to RFA were observed during, after, and at the moment of surgical resection in the delayed group. Postoperative hospital stay was uneventful.

In all cases the thermal lesion encompassed the tumor. On macroscopic examination it appeared as rounded bloodless necrotic tissue with a diameter of $4.1 \mathrm{~cm}$ on average (range $2.9-5.3 \mathrm{~cm}$ ). A central cavitation was surrounded by white coagulated tissue, partially carbonized in some cases. In patients who underwent immediate surgical resection, a peripheral reddish rim was well demarcated from both the central coagulated tissue and surrounding normal-appearing parenchyma. Lesions observed in patients who underwent percutaneous RFA and delayed resection were better demarcated, and a thin rim of light fibrous scar tissue was observed at the periphery of the lesion, replacing the red damaged area (Figure 2). Microscopic examination showed the presence of coagulation necrosis. In particular, the cytoplasm lost its structural detail (eg, granularity and striations) and resembled coagulated material, thus the term "coagulative necrosis." The cytoplasm was more eosinophilic (pinkish red) because of loss of hematoxylin-binding ribosomes and increased affinity for eosin. The nucleus shrinks irregularly and stains more deeply (pyknosis), breaks up into fragments (karyorrhexis), and finally fails to stain with hematoxylin (karyolysis). This is caused by the action of released lysosomal enzymes, that is, proteases, exonucleases, and phosphatases. No viable tissue was visible within the necrotic area, except at its extreme periphery where some nests of viable cells persisted, particularly along the bigger vessels. Early inflammation cells, edema, and damaged alveoli were seen within $1 \mathrm{~cm}$ peripherally of the necrotic nodule. However, no injury was noted within this peripheral area in blood vessels and bronchi, except for a dilatation of smaller vessels. No anatomopathologic alterations were seen in the surrounding distant parenchyma ( $\geq 2 \mathrm{~cm}$ from the periphery of the necrotic area). The only difference in the specimens

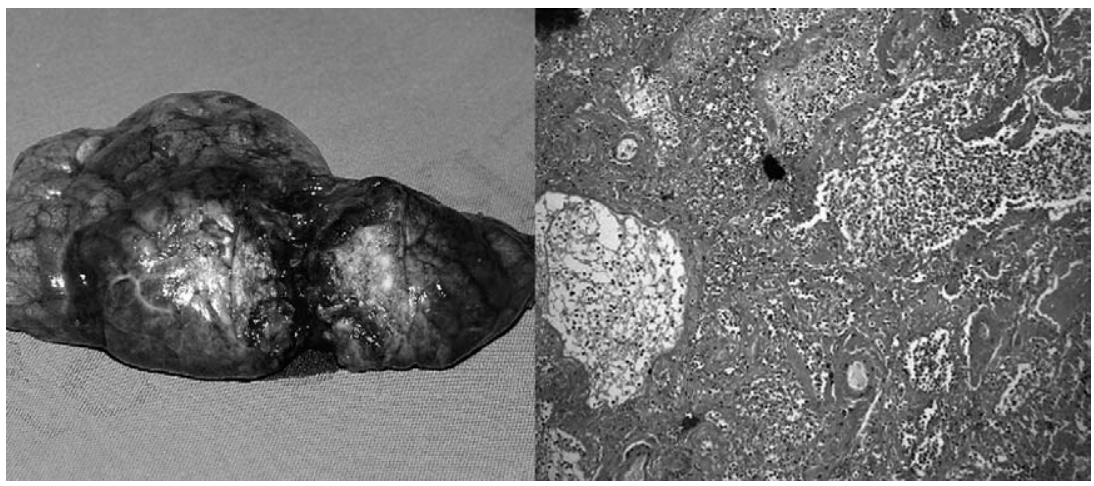

Figure 2. Macroscopic and microscopic reports of an NSCLC resected 15 days after percutaneous CT-guided RFA showing complete coagulation necrosis. 
obtained 15 days after RFA was the presence of granulation tissue surrounding the necrotic area, with the inflammatory cells that were cleaning up damaged tissue and leading scar tissue synthesis. Still, no damage was observed in the parenchyma surrounding the thermal lesion.

Complete necrosis of the tumor was recorded in 6 of 9 cases (Figure 2). In these patients the mean size of the neoplastic lesion was $2.3 \mathrm{~cm}$. Clusters of viable but injured tumor cells were observed in 3 patients, principally at the periphery of the lesion ( 2 patients who underwent immediate resection and 1 patient who underwent delayed resection). The clusters represented no more than $10 \%$ of the treated area and, in many cases, appeared completely surrounded by necrotic tissue, probably without blood supply. Even if the thermal lesion completely encompassed the tumor, its margins from the neoplastic lesion appeared shorter ( $<5 \mathrm{~mm}$ in all 3 cases) than in the patients with complete ablation ( $8 \mathrm{~mm}$ on average). Moreover, the mean size of the lesions in case of incomplete ablation was 3.1 $\mathrm{cm}$, larger than that of those completely ablated but without statistical significance. There were no other significant differences between the group with complete necrosis and the group with incomplete ablation.

The patient who refused surgical resection after percutaneous RFA is still alive and locally disease-free at a follow-up period of 33 months. A second tumor recently developed in his contralateral lung, which was still treated by RFA.

\section{Discussion}

Since thermal ablation of a pulmonary tumor using radiofrequency was first achieved by Lilly and colleagues in $1983,{ }^{13}$ much has been learned. Currently, many articles report promising clinical results in performing percutaneous thermal ablation of primary or secondary lung tumors. ${ }^{8-11}$ Notwithstanding that in recent years a great amount of data on the clinical and radiologic response of RFA on lung tumors have accumulated, its biologic effects remain poorly understood.

The first "ablate and resect" model of pulmonary neoplasms was described by Goldberg and colleagues in $1996^{14}$ on an animal model with a pulmonary tumor induced by means of injection of a suspension of sarcomatose cells VX2. Seven rabbits, each with a tumor 6 to $12 \mathrm{~mm}$ in diameter, were treated for 6 minutes at $90^{\circ} \mathrm{C}$. The anatomopathologic examination, executed at several intervals of time after treatment, showed the presence of a necrotic area with absence of neoplastic cells in $95 \%$ of the cases.

Steinke and colleagues, ${ }^{15}$ in 2002, published the first article describing an "ablate and resect" protocol in a single patient. They performed CT-guided radiofrequency ablation of a pulmonary metastasis followed by surgical resection. Histologic workup, 3 weeks postablation, showed complete devitalization of the metastasis. An abstract by Scott and colleagues, ${ }^{16}$ pre- sented at the 10th World Conference on Lung Cancer in 2003, reported a $100 \%$ of necrosis in 4 of 5 patients (80\%). Our experience confirms and strengthens their findings. As expected, as the number of cases increased, more failures of the procedure were observed. In 3 of 9 cases, clusters of viable tumor cells were observed. They represented no more than $10 \%$ of the treated area and appeared injured and completely surrounded by necrotic tissue, probably without blood supply. The same authors recently published an extension of the study. ${ }^{17}$ They reported $100 \%$ necrosis in 3 of 8 patients $(37.5 \%)$ and more than $80 \%$ tumor necrosis in 4 of 5 of the remaining patients. They used supravital staining (reduced nicotinamide adenine dinucleotide [NADH]), maintaining that it "was the only histopathologic method capable of differentiating viable from ablated tissue," yet without supporting the statement with references. We also discussed the opportunity to use this method with our pathologist and decided not to use $\mathrm{NADH}$ staining. The method is very delicate and requires a procedure that we were not able to reproduce correctly, primarily because our thoracic surgery unit and pathology unit are located in 2 different hospitals. Nguyen and colleagues ${ }^{17}$ excluded a patient because of an incorrectly performed supravital stain. Moreover, even if NADH staining could supply some more details about cell viability in tumor cells that are not clearly dead, necrosis is always well assessed by standard hematoxylin-eosin staining. ${ }^{18}$ Their results, worse than those of our experience despite NADH staining, could be explained with the differences in the 2 pilot studies. In fact, even if the mean size of the lesions was similar (2.2 vs $2.8 \mathrm{~cm}$ ), they used different technology and RFA protocols. Their device to bring radiofrequency energy into the tumor had a single electrode of 3 or $3.5 \mathrm{~cm}$; our needle had 9 electrodes that were deployable in a flower-like manner for $5 \mathrm{~cm}$, which allows better encompassment of the tumor. Moreover, the mean time of RFA was almost double in our series. Both of these aspects may justify the better results in our experience. Our "ablate and resect" protocol reports an overall success rate of RFA in local eradication of NSCLC of approximately 67\%, which is similar to those recently reported in clinical trials. ${ }^{10,11}$ When wedge resection is used, it is important to obtain wide margins between the tumor and the staple line. It is not surprising that in the cases in which the tumor was not completely ablated, the margins of ablation were smaller.

Another aspect underlined and strengthened in this study was the absence of damage in peripheral lung parenchyma more than $1 \mathrm{~cm}$ distant from the thermal lesion. The thermal lesion, in fact, seems to limit itself in the lung, probably because of ventilation and blood supply of normal parenchyma. We $\mathrm{W}^{12}$ and Goldberg and colleagues ${ }^{19}$ have reported such encouraging phenomenon in previous articles on animal models. In both experiences, the most important aspect was precisely this auto-limitation of the thermal injury itself, which did not affect the peripheral parenchyma. Furthermore, Gold- 
berg and colleagues observed that the dimensions of the lesion induced in pulmonary parenchyma were lower than those induced in other solid organs like the liver. They related these facts to the high impedance of the inflated lung that reduces propagation of radiofrequency waves. This behavior could prove to be useful in RFA of solid pulmonary neoplasms. In fact, the energy would be adequately propagated in the solid tissue, obtaining coagulation and necrosis, while the surrounding healthy parenchyma would be protected from air inflation with a kind of physiologic auto-delimitation of the thermal damage. Such a supposition has been confirmed in this study. The thermal lesions were on average $1.3 \mathrm{~cm}$ larger than the neoplastic ones and were well-demarcated from the surrounding parenchyma, which showed no macroscopic or microscopic alterations $1 \mathrm{~cm}$ or more distant from the periphery of the necrotic area.

\section{Conclusion}

The feasibility and safety of RFA of lung tumors have been recently assessed in several articles in the literature, whereas its efficacy still remains to be determined. This study confirmed the safety of the procedure, particularly underlining the possibility of performing a thermal lesion in the lung parenchyma in a controlled manner, but, above all, the capability of RFA to produce complete necrosis of a lung tumor, as it was histologically proven in $67 \%$ of patients in our series. A limitation of this pilot study is the number of the enrolled patients. Even if, to our knowledge, this is the largest reported series, it is required that other similar studies confirm our results. Certainly it is necessary to underline the purpose of RFA, which is only the local treatment of the tumor, with all its limits. In fact, when wedge resection is compared with lobectomy, when we treat a lung tumor with RFA instead of surgical resection, despite a successful procedure, a high recurrence rate is expected. Well-designed clinical trials, with long-term follow-up, are required to confirm the preliminary results before RFA can enter into clinical practice. However, since wedge resection is considered a compromise operation with respect to lobectomy, then RFA must be considered a compromise procedure with respect to surgical resection in high-risk patients.

\section{References}

1. Van Schil PE. Surgery for non-small cell lung cancer. Lung Cancer. 2001;34(Suppl 2):S127-32.

2. Zimmermann FB, Bamberg M, Molls M, Jeremic B. Radiation therapy alone in early stage non-small cell lung cancer. Semin Surg Oncol. 2003;21:91-7.

3. Lencioni R, Goletti O, Armillotta N, et al. Radio-frequency thermal ablation of liver metastases with a cooled-tip electrode needle: results of a pilot clinical trial. Eur Radiol. 1998;8:1205-11.

4. Rosenthal DI, Springfield DS, Gebhardt MC, Rosenberg AE, Mankin HJ. Osteoid osteoma: percutaneous radio-frequency ablation. Radiology. 1995; 197:451-4.

5. Wood BJ. Feasibility of thermal ablation of lytic vertebral metastases with radiofrequency current. Cancer J. 2002;8:26-9.

6. Goldberg SN, Wood BJ, Gervais DA, et al. Radiofrequency ablation of renal cell carcinoma: early clinical experience. Radiology. 2000;217: 665-72.

7. Anzai Y, Lufkin R, Desalles A, et al. Preliminary experience with a MR-guided thermal ablation of brain tumor. AJNR Am J Neuroradiol. 1995;16:39-48.

8. Dupuy DE, Zagoria RJ, Akerley W, Mayo-Smith WW, Kavanagh PV, Safran H. Percutaneous radiofrequency ablation of malignancies in the lung. Am J Roentgenol. 2000;174:57-9.

9. Herrera LJ, Fernando HC, Perry Y, et al. Radiofrequency ablation of pulmonary malignant tumors in non surgical candidates. $J$ Thorac Cardiovasc Surg. 2003;125(4):929-37.

10. Fernando HC, De Hoyos A, Landreneau RJ, et al. Radiofrequency ablation for the treatment of non-small cell lung cancer in marginal surgical candidates. J Thorac Cardiovasc Surg. 2005;129:639-44.

11. Ambrogi MC, Lencioni R, Fontanini G, et al. Percutaneous radiofrequency ablation of primary NSCLC. Lung Cancer. 2005;49(Suppl 2):S3.

12. Ambrogi MC, Fontanini G, Lencioni R, et al. A preliminary study on thermal ablation of lung tumour. Contemp Oncol. 2004;8:373-9.

13. Lilly MB, Brezovich IA, Atkinson W, et al. Hyperthermia with implanted electrodes: in vitro and in vivo correlations. Int J Radiat Oncol Biol Phys. 1983;9:373-82.

14. Goldberg SN, Gazelle GS, Compton CC, Mueller PR. Radiofrequency tissue ablation of VX2 tumor nodules in the rabbit lung. Acad Radiol. 1996;3:929-35.

15. Steinke K, Habicht JM, Thomsen S, Soler M, Jacob AL. CT-guided radiofrequency ablation of a pulmonary metastasis followed by surgical resection. Cardiovasc Intervent Radiol. 2002;25:543-6.

16. Scott WJ, Young N, Goldberg M, Langer C, Movsas B, Rogatkp A Tumor cell viability following radiofrequency ablation of resectable primary lung cancer: initial results from an ablate and resect study. Lung Cancer. 2003;41(Suppl 2):135.

17. Nguyen CL, Scott WJ, Young NA, Rader T, Giles LR, Goldberg M Radiofrequency ablation of primary lung cancer. Results from an ablate and resect pilot study. Chest. 2005;128:3507-11.

18. Jeffrey SS, Birdwell RL, Ikeda DM, et al. Radiofrequency ablation of breast cancer: first report of an emerging technology. Arch Surg. 1999; $134: 1064-8$.

19. Goldberg SN, Gazelle GS, Compton CC. Radiofrequency tissue ablation in the rabbit lung: efficacy and complications. Acad Radiol. $1995 ; 2: 776-84$. 